\title{
Correlating the Entropy of a Fluid with Live Collective Behaviors $^{+}$
}

\section{Edgar Olivares Mañas, and Richard P Donovan}

University of California Irvine, Irvine, CA, USA

+ Presented at the Entropy 2021: The Scientific Tool of the 21st Century, 5-7 May 2021; Available online: https://sciforum.net/conference/Entropy2021/.

Published: 5 May 2021

Given a group of individuals inside a fluid flow (e.g., air or water) that behaves as a collective to some degree, factors like the shape of a group or the distance between its individuals can affect the forces that each individual inside the group may notice, such as drag or lift forces. Bird flocks, swarms or cycling riders are just some group behavior examples where the topology of fluid flow and information exchange affects the aerodynamics of the whole group, and may also interact with other groups or external individuals.

This group dynamics and interaction can be numerically correlated by entropy. The fundamental basis of the most of Computational Fluid Dynamics (CFD) problems is the Navier-Stokes (NS) equations, which define many single-phase fluid flows with some multi-phase flow extensions. NS equations are written as a function of the fluid velocity and pressure (in addition to other variables that describe the fluid properties), but they can also be re-written as a function of entropy. However, it is not easy to find a direct methodology that relates the entropy of a system, the fluid dynamics and the collective behavior of a system inside this fluid.

In this work we propose a method to easily calculate the entropy of a CFD solution by computing the pixels of its image based on Shannon entropy. Using the results obtained, we study the relation between information mechanics, fluid dynamics and fluid forces.

(C) 2021 by the authors. Licensee MDPI, Basel, Switzerland. This article is an open access article distributed under the terms and conditions of the Creative Commons Attribution (CC BY) license (http://creativecommons.org/licenses/by/4.0/). 